\title{
A DIFERENCIAÇÃO PEDAGÓGICA NO CONTEXTO DA EDUCAÇÃO INCLUSIVA
}

\author{
Vítor Ochoa Maial \\ Sofia Freire ${ }^{2}$
}

\section{RESUMO}

A diferenciação pedagógica é uma abordagem ao ensino em sala de aula que tem sido apontada como relevante na promoção da participação e sucesso de todos os alunos. É, por essa razão, muitas vezes descrita como uma componente essencial das práticas inclusivas. Contudo, apesar das evidências positivas do seu efeito no desempenho dos alunos, quer em termos acadêmicos, quer em termos pessoais, os professores tendem a implementá-la pouco. Uma das razões apontadas para que os professores não desenvolvam frequentemente práticas de diferenciação pedagógica na sala de aula é a falta de clarificação desse conceito, que deu origem a uma representação desalinhada dos princípios da diferenciação pedagógica inclusiva. Assim, torna-se importante esclarecer o que se entende, de fato, por diferenciação pedagógica no contexto da educação inclusiva. Para esse efeito, será apresentado o modelo de diferenciação pedagógica segundo a proposta de Tomlinson (2008), por configurar uma visão mais integrada e explícita da diversidade em sala de aula, como pressupõe a diferenciação pedagógica inclusiva. Simultaneamente, serão apontadas as potencialidades do seu desenvolvimento e algumas barreiras que têm sido identificadas à sua implementação em sala de aula. Espera-se, assim, dar um contributo para uma reflexão sobre as práticas dos professores e equacionar caminhos para a sua eventual mudança.

Palavras-chave: Diferenciação Pedagógica. Diversidade. Educação Inclusiva.

\section{PEDAGOGICAL DIFFERENTIATION IN THE CONTEXT OF INCLUSIVE EDUCATION}

\begin{abstract}
Pedagogical differentiation is an approach to teaching in the classroom that has been pointed out as relevant in promoting the participation and success of all students. It is for this reason often described as an essential component of inclusive practices. However, despite the positive evidence of its effect on student performance, both in academic and personal terms, teachers tend to implement it

1 Mestrado. Agrupamento de Escolas Daniel Sampaio, Almada, Portugal. Orcid iD: https://orcid.org/0000-0001-5571-6178. E-mail: vitor.maia@campus.ul.pt

2 Doutorado. Instituto de Educação da Universidade de Lisboa, Portugal. Orcid iD: https://orcid.org/0000-0002-9394-9738. E-mail: asraposo@ie.ulisboa.pt
\end{abstract}


little. One of the reasons pointed out that teachers often do not develop practices of pedagogical differentiation in the classroom is the lack of clarification of this concept, which gave rise to a misaligned representation of the principles of inclusive pedagogical differentiation. Thus, it becomes important to clarify what is understood, in fact, by pedagogical differentiation in the context of inclusive education. To this end, the model of pedagogical differentiation according to Tomlinson's proposal (2008) will be presented, as it will provide a more integrated and explicit view of diversity in the classroom, as it presupposes inclusive pedagogical differentiation. At the same time, the potentialities of its development and some barriers that have been identified to its implementation in the classroom will be pointed out. It is hoped, therefore, to contribute to a reflexion on the practices of the teachers and to find ways for their eventual change.

Keywords: Pedagogical Differentiation. Diversity. Inclusive Education.

\section{LA DIFERENCIACIÓN PEDAGÓGICA EN EL CONTEXTO DE LA EDUCACIÓN INCLUSIVA}

\section{RESUMEN}

La diferenciación pedagógica es un enfoque a la enseñanza en el aula que se ha señalado como relevante en la promoción de la participación y el éxito de todos los alumnos. Por lo tanto, a menudo se describe como un componente esencial de las prácticas inclusivas. Sin embargo, a pesar de las evidencias positivas de su efecto en el desempeño de los alumnos, tanto en términos académicos como personales, los profesores tienden a implementarla poco. Una de las razones apuntadas para que los profesores no desarrollen a menudo prácticas de diferenciación pedagógica en el aula es la falta de clarificación de ese concepto, que dio origen a una representación desalineada de los principios de la diferenciación pedagógica inclusiva. Así, es importante aclarar lo que se entiende, de hecho, por diferenciación pedagógica en el contexto de la educación inclusiva. Para ello, se presentará el modelo de diferenciación pedagógica según la propuesta de Tomlinson (2008), por configurar una visión más integrada y explícita de la diversidad en el aula, como presupone la diferenciación pedagógica inclusiva. Simultáneamente, se señalarán las potencialidades de su desarrollo y algunas barreras que se han identificado a su implementación en el aula. Se espera, así, contribuir a una reflexión sobre las prácticas de los profesores y abordar caminos para su eventual cambio.

Palabras clave: Diferenciación Pedagógica. Diversidad. Educación Inclusiva.

\section{INTRODUÇÃO}

A educação inclusiva é uma perspectiva que assume que a educação é para todos (EASNIE, 2018) e que o sistema educativo deve fortalecer-se continuamente para alcançar todos os alunos, i.e., deve ser capaz de identificar, a cada momento, as barreiras que impedem a 
participação e a aprendizagem de todos os alunos e procurar encontrar o modo de as superar (UNESCO, 2017). Esse processo contínuo de fortalecimento da capacidade do sistema educativo para responder a todos pressupõe educar a diversidade dos alunos (SANCHES, 2005; UNESCO, 2009). É esse o desafio que os professores têm de enfrentar na sala de aula e que exige equacionar abordagens ao ensino, que não sejam uniformizadas e que atendam às diferenças de todos.

É neste contexto que surge a diferenciação pedagógica inclusiva como uma abordagem ao ensino que promove a inclusão e a equidade educativa (SOUSA, 2010). Contudo, apesar das evidências positivas do seu potencial na promoção da participação e sucesso de todos os alunos na sala de aula (ROCK et al., 2008; TIESO, 2002; UNESCO, 2017), os professores tendem a não a implementar como uma prática incorporada nas suas rotinas de trabalho diárias (CIVITILLO et al., 2016; PRUD'HOMME et al., 2005; TOMLINSON et al., 2003; TOMLINSON, 2016). Essa situação deve-se, em parte, aos professores não terem uma noção clara do que seja a diferenciação pedagógica ou terem uma noção imprecisa do que ela é (FEYFANT, 2016; PRUD'HOMME et al., 2016), julgando, neste caso, estar a desenvolver práticas de diferenciação pedagógica quando, na realidade, não estão.

Não sendo um conceito novo, a sua evolução acompanhou diversas denominações e diferentes perspectivas teóricas que as enformaram (PRUD'HOMME et al., 2016), sendo uma delas a diferenciação pedagógica como ensino individualizado, a sua primeira acepção (CORNO; SNOW, 1986). Desta associação conceptual resultou uma representação, que se cristalizou com os professores e nas escolas, em que a diferenciação pedagógica é uma abordagem ao ensino orientada apenas para os alunos que, por razões variadas, não conseguem acompanhar a aula padrão, comum a todos. Essa forma de compreender a diferenciação pedagógica encontra-se desalinhada dos princípios da diferenciação pedagógica inclusiva, tal como defendido por Tomlinson (2014).

Para além da imprecisão conceptual, os professores tendem a implementar pouco essas práticas em razão das suas crenças sobre 
diferenciação pedagógica, da falta de formação, do modo como concebem a diversidade, da falta de tempo, entre outras (e.g., CIVITILLO et al., 2016; DIXON et al., 2015; HEHIR et al., 2016; JAGER, 2013; WAN, 2017). É, por isso, importante clarificar para professores e escolas o que é a diferenciação pedagógica inclusiva e apoiá-los na reflexão de suas práticas e no equacionamento da utilização de outras mais, consonantes com o potencial para responder à diversidade.

Face ao exposto, este artigo tem como principal objetivo clarificar o conceito de diferenciação pedagógica, como promotora de uma educação inclusiva, à luz do modelo apresentado por Tomlinson (2008). Para esse efeito, começar-se-á pelo esclarecimento do que é a diferenciação pedagógica no contexto da educação inclusiva; na sequência, tratar-se-á da imprecisão conceptual gerada ao longo da sua evolução, de modo a mostrar que tal imprecisão deu origem a uma representação que os professores criaram sobre a diferenciação pedagógica e que essa representação os tem impedido de desenvolver práticas alinhadas com os princípios da diferenciação pedagógica inclusiva; finalizar-se-á com a identificação das potencialidades do seu desenvolvimento em sala de aula e de algumas barreiras à sua implementação, juntamente com sugestões para que os professores possam equacionar eventuais caminhos de mudança.

\section{DIFERENCIAÇÃO PEDAGÓGICA INCLUSIVA}

O tema da inclusão na educação tem sido uma prioridade de muitos países (ANGELIDES, 2008) e um foco de considerável atividade nos últimos anos (MESSIOU et al., 2016), sendo para EASNIE (2018) a abordagem para a educação no século XXI. Nas últimas duas décadas, muitos esforços têm sido empreendidos para promover a educação inclusiva através de compromissos internacionais, assumidos pelos governantes, e para promover políticas e práticas educacionais mais alinhadas com os princípios orientadores da educação inclusiva, o que resultou em alguns progressos (UNESCO, 2017). Ainda assim, é necessário continuar a envidar caminhos 
para que os alunos experimentem um ambiente genuinamente inclusivo (UNESCO, 2017), que assegure o sucesso de todos e de cada um (SANCHES; TEODORO, 2007).

A educação inclusiva assume que a educação é para todos (EASNIE, 2018; LATORRE, 2013; SANCHES, 2005), em que todos aprendem juntos no grupo e com o grupo heterogêneo (SANCHES, 2005), dentro da sala de aula (SANCHES; TEODORO, 2007). Neste sentido, há uma preocupação com a superação das barreiras que impedem a participação e a aprendizagem de todos os alunos (ANGELIDES, 2008; LATORRE, 2013), o que pressupõe o desenvolvimento de culturas, políticas e práticas inclusivas nos sistemas educacionais e escolares (ANGELIDES, 2008; AINSCOW; BOOTH, 2002; LATORRE, 2013).

A educação inclusiva é um processo contínuo (AINSCOW, 2009; ANGELIDES, 2008) de fortalecimento da capacidade do sistema educacional para responder a todos e a cada um dos alunos (UNESCO, 2009; 2017), reconhecendo as suas diferenças individuais como oportunidades para enriquecer a aprendizagem e beneficiá-los a todos (UNESCO, 2017). Nesse sentido, falar de educação inclusiva é falar sobre a responsabilidade da escola incluir e educar a diversidade dos alunos (SANCHES, 2005; UNESCO, 2009) e procurar gerar o sucesso de todos através do sucesso de cada um (SANCHES; TEODORO, 2007). Isso implica conceber a diversidade como condição humana (LATORRE, 2013), como "O direito de cada indivíduo ser considerado de acordo com as suas experiências, conhecimentos anteriores, motivações e estilos de aprendizagem" (SÁNCHEZ, 2009, p. 90).

Nas últimas décadas, a diversidade de alunos tornou-se uma questão relevante no discurso educacional (KALDI, GOVARIS; FILIPPATOU, 2018). Essa diversidade em sala de aula manifesta-se muito além dos resultados acadêmicos dos alunos (FERREIRA, 2017), sendo visível nas suas diferenças de interesses, de estilos de aprendizagem, de background cultural e educacional, de ritmos de aprendizagem, de competências educativas (CIVITILLO et al., 2016; DIXON et al. 2014; FEYFANT, 2016; KALDI, GOVARIS; FILIPPATOU, 2018; PARSONS, DODMAN; BURROWBRIDGE; 2013; TOMLINSON, 
2008) e mesmo nas relações interpessoais, na comunicação verbal e nãoverbal e na motricidade (FERREIRA, 2017).

Contudo, responder à diversidade em sala de aula de forma a proporcionar a todos os alunos uma aprendizagem eficaz e promover, simultaneamente, a sua participação e o seu sucesso (UNESCO, 2017), é um desafio complexo, exigente e difícil (CIVITILLO et al., 2016; DIXON et al., 2014; JAGER, 2013; ROCK et al., 2008; TOMLINSON et al., 2003), que implica uma conceção de organização e gestão escolar que seja capaz de reconhecer o direito à diferença (FERREIRA, 2017) e de considerar essa diversidade como um fator de enriquecimento e desenvolvimento pessoal e acadêmico (CORREIA, 2001; LIMA-RODRIGUES, 2007; UNESCO, 2005). No campo de ação dos professores, é necessário que desenvolvam práticas diferenciadas de ensino em sala de aula (TOMLINSON et al., 2003).

A diferenciação pedagógica é descrita muitas vezes como uma componente essencial das práticas inclusivas (AINSCOW; BOOTH, 2002), pois procura assegurar que todos os alunos, independentemente das suas diferenças, conseguem ter sucesso na aprendizagem (FEYFANT, 2016; UNESCO, 2017). É, nesse sentido, que Sousa (2010, p.15) se refere à diferenciação como "um instrumento de promoção da inclusão e da equidade face às diferenças existentes no seio da população estudantil".

\section{Diferenciação pedagógica e individualização: uma representação confusa}

Muito embora a diferenciação pedagógica seja um assunto que está no centro da agenda política portuguesa (e.g. Decreto-Lei no 54/2018 de 6 de julho), esse conceito é já antigo. De fato, as primeiras referências à diferenciação pedagógica, enquanto ensino adaptado às características individuais do aluno para promover a sua aprendizagem e o seu sucesso educativo, datam do século IV a.C., na China, no Tratado Xue Jie de Yuezheng, tal como na antiga Hagadá de Passach hebraica e no século I d.C, em Roma, no De Institutione Oratoria de Quintiliano (CORNO; SNOW, 1986). 
Nos séculos subsequentes, esse conceito evoluiu em linha com diversas perspectivas teóricas que se sucederam e, por vezes, se sobrepuseram, nomeadamente entre uma concepção individual da diferenciação (e.g., Parkhurst, cit. MEIRIEU, 2013) e uma coletiva ou comunitária (e.g., Faure, cit. MEIRIEU, 2013; PRUD'HOMME et al., 2016). Simultaneamente, diferentes denominações foram surgindo, tanto no mundo francófono como no anglófono, e.g., diferenciação pedagógica, pedagogia diferenciada, pedagogia variada, ensino diferenciado, individualização, etc., sem que os seus aspectos distintivos fossem clarificados com rigor, situação que gerou uma imprecisão conceptual à volta da noção de diferenciação (PRUD'HOMME et al., 2016).

Uma das consequências desta ambiguidade foi a confusão que se estabeleceu entre individualização e diferenciação, "quase como se uma fosse a consequência da outra" (FEYFANT, 2016, p. 7, tradução livre), o que consolidou uma percepção (nas escolas e, em particular, nos professores) de que, apesar das suas variantes, a diferenciação seria um modo de remediação no decorrer da aula, uma resposta para os alunos que se "desviam" da aula padrão, comum a todos, e uma espécie de ensino individualizado, i.e., um meio de responder às dificuldades específicas dos alunos escolarmente mais fracos (FEYFANT, 2016), muito à semelhança do pensamento e da prática da educação especial (UNESCO, 2017).

Esta ideia de diferenciação pedagógica, como um ensino individualizado, uma adaptação e um ajustamento pontuais, orientados para alunos específicos, "perdeu terreno" após os anos setenta do século XX, com a exigência de democratização do ensino e da escola. Com efeito, uma visão mais abrangente da diferenciação pedagógica, distante do modelo de individualização, ganhou relevância, face às tentativas frustradas para reduzir as desigualdades escolares e os efeitos diferenciadores da escola (FEYFANT, 2016).

Nesse contexto, tornou-se necessário encontrar um caminho para ensinar um mesmo programa em turmas muito heterogêneas e para lutar contra o insucesso escolar que era cada vez menos aceito socialmente 
(ROBBES, 2009), o que parecia ser possível com a mobilização de uma diversidade de metodologias disponíveis para todos os alunos e não apenas para um conjunto de alunos que, por razões diversas, não atingiam os objetivos esperados para o seu nível, de acordo com um modelo de aluno "médio" definido implicitamente pelo estado (FERREIRA, 2017). No entanto, este entendimento da diferenciação, como resposta à heterogeneidade dos alunos em sala de aula e como um meio de promover o seu sucesso escolar, não conseguiu dissociar-se, de modo evidente, da concepção de individualização, pois os dispositivos de diferenciação continuaram a serviço dos alunos que não conseguiam acompanhar o grupo por dificuldades e lacunas graves (PRZESMYCKI, 1991), fazendo perpetuar a confusão entre individualização e diferenciação.

Essa imprecisão conceptual reforçou a representação que os professores já tinham construído deste conceito (FEYFANT, 2016; PRUD'HOMME et al., 2016), encarando-o, fundamentalmente, como um meio de atenuar as dificuldades dos alunos com necessidades de aprendizagem específicas; como um modo de resposta, no decorrer da aula, aos alunos que "saíam" da mesma lição que era ensinada a todos; como um modo de individualização ou reagrupamento contínuo dos alunos, segundo níveis de desempenho fracos ou fortes. Assim, representada, a diferenciação pedagógica levou os docentes, muitas vezes, a "afastarem-se dos saberes coletivos, comuns à classe" (FEYFANT, 2016, p. 2, tradução livre) e contribuiu para o seu sentimento de impotência relativamente à diversidade encontrada na sala de aula (PRUD'HOMME et al., 2016). Por essa razão, alguns autores referem que é urgente clarificar o conceito de diferenciação pedagógica com os professores para: (i) facilitar o reconhecimento da sua relação com a valorização da diversidade presente em sala de aula (FERREIRA, 2017); (ii) compreender, de forma situada e dinâmica (PRUD'HOMME et al., 2016), a complexidade do trabalho associado à mesma; e (iii) favorecer a transição das intenções à ação (HUMPHREY et al., 2006; TOMLINSON et al., 2003). 


\section{Princípios orientadores da diferenciação pedagógica inclusiva}

Dentre as várias orientações conceptuais que marcaram o desenvolvimento da diferenciação pedagógica (PRUD'HOMME et al., 2016), encontra-se a perspectiva de Tomlinson (2008) que configura uma visão mais integrada e explícita da diversidade em sala de aula e que, por essa razão, responde de forma adequada à ideia subjacente à diferenciação pedagógica inclusiva, pois permite aos alunos aprenderem a reconhecer ou a descobrir as diferenças entre si e a captar o valor e a riqueza da mesma para o grupo (PRUD'HOMME et al., 2016). Como referem Prud'homme e colaboradores (2016), "O professor que diferencia num contexto inclusivo encontrará diferentes meios para apoiar e encorajar a expressão da diversidade, assim como para colocar em evidência a contribuição que estas diferenças podem trazer ao processo de ensino-aprendizagem" (p. 127, tradução livre).

Para se perceber plenamente $\bigcirc$ modelo de diferenciação pedagógica apresentado por Tomlinson (2008), é fundamental reconhecer, primeiramente, que a diferenciação se realiza pela forma como se percebe e se pensa a educação (FEYFANT, 2016), que ela é tanto uma filosofia (que pressupõe a adoção de certos valores e atitudes) como um modelo orientado por um conjunto de princípios e práticas que visam um ensino e uma aprendizagem efetivos (HOCKETT, 2018; TOMLINSON; IMBEAU, 2010). Nesse sentido, é importante que os professores compreendam esses princípios para poderem, a partir deles, desenvolver os seus próprios métodos (TOMLINSON; IMBEAU, 2010) e aplicarem, de forma refletida e consistente, a diferenciação na sala de aula.

A diferenciação pedagógica é orientada pelos seguintes princípios gerais (Figura 1): ambiente de aprendizagem positivo (positive learning environment), currículo de alta qualidade (high-quality curriculum), avaliação contínua (ongoing assessment), ensino orientado para as necessidades dos alunos (instruction that responds to student needs) e sala de aula flexível (flexible classroom) (TOMLINSON; IMBEAU, 2010; TOMLINSON, 2014; SOUSA; TOMLINSON, 2018). 
FIGURA 1 - Princípios orientadores da diferenciação pedagógica

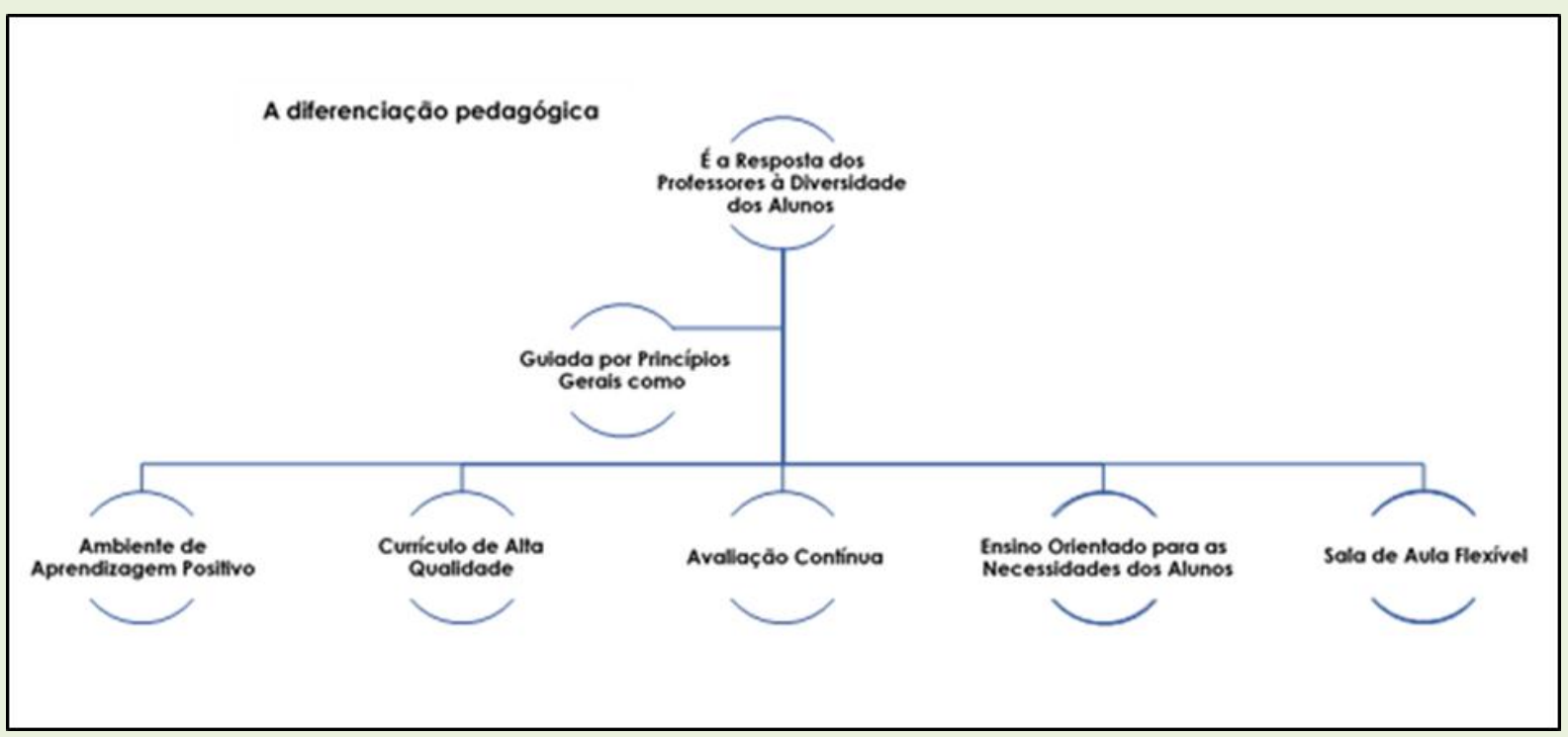

Fonte: Os autores.

Uma aula de ensino diferenciado proporciona um ambiente de aprendizagem positivo. Esse ambiente de aprendizagem, como o contexto físico e emocional em que a aprendizagem ocorre, está associado à organização e estrutura de uma sala de aula, que deve convidar todos os alunos à aprendizagem, i.e., a investirem os seus melhores esforços para aprenderem total e profundamente (SOUSA; TOMLINSON, 2018), exibindo, por exemplo, alguns dos seus trabalhos, facilitando o acesso a materiais, dispondo o mobiliário para que promova a colaboração, entre outros aspectos. Para além disso, é importante que os alunos se sintam seguros, respeitados, envolvidos, desafiados e apoiados, pois, dessa forma, aprendem melhor (TOMLINSON; IMBEAU, 2010).

O currículo diz respeito àquilo que é aprendido e ensinado, ao modo como são concretizados, à avaliação e à utilização dos recursos/materiais. Os professores tendem a considerar o currículo formal como um conjunto de resultados e metas educacionais prescritos por uma autoridade, e que são constrangidos a implementá-lo rigidamente (UNESCO, 2004). Contudo, há igualmente o currículo informal ou o currículo "escondido" - a aprendizagem não planejada que ocorre na sala de aula, na escola e nas relações entre alunos, com ou sem a presença dos professores. É importante estar ciente 
desse currículo, pois pode ser usado para reforçar a aprendizagem formal (UNESCO, 2004). De acordo com Tomlinson e Imbeau (2010), o currículo é um plano organizado para envolver os alunos com saberes (knowledge), conhecimentos (understanding) e competências (skills) importantes. Um currículo de alta-qualidade pressupõe um bom conhecimento, por parte do professor, da natureza da disciplina, que o currículo representa, e uma definição clara do que os alunos devem saber e das competências e conhecimentos que devem desenvolver como resultado de um segmento de aprendizagem (e.g., um ano, uma unidade de estudo, um módulo, um tema). Inclui uma sequência de aulas planejadas cuidadosamente (TOMLINSON; IMBEAU, 2010), com grande relevância para os alunos e possibilitando grande envolvimento dos mesmos (SOUSA; TOMLINSON, 2018).

A avaliação é extraordinariamente importante quando se pretende dinamizar aulas de ensino diferenciado; é o que garante a sua eficácia. Pela avaliação, o professor consegue recolher informação sobre as características dos alunos, planificar as suas aulas de acordo com ela e fazer os ajustes necessários no decorrer das mesmas. É nesse sentido que Sousa e Tomlinson (2018) consideram a avaliação contínua, processo regular de recolha e análise de informação que permite ao professor ir modificando os seus planos de aula, de acordo com os pontos fortes e fracos dos alunos, com o objetivo de ajudá-los a crescer academicamente.

A avaliação pode ser diagnóstica, formativa e somativa. No primeiro caso, serve para conhecer previamente o nível de preparação do aluno relativamente aos resultados das aprendizagens essenciais quando uma unidade de estudo inicia, bem como os seus interesses e perfil de aprendizagem. No segundo caso, permite acompanhar de forma constante o progresso de um aluno, possibilitando ao professor ajudá-lo a compreender qual o melhor caminho a seguir. No terceiro caso, permite medir os resultados do aluno quando a unidade de estudo termina ou num outro momento chave de uma unidade ou de um ano (TOMLINSON; IMBEAU, 2010; SOUSA; TOMLINSON, 2018). 
A par da avaliação, como orientadora do processo educativo, e alinhado com ela, o ensino deve ser orientado para responder às diferentes necessidades de todos os alunos, providenciando diversas abordagens à aprendizagem (SOUSA; TOMLINSON, 2018). Nesse sentido, por exemplo, as tarefas propostas devem ser igualmente importantes para todos, proporcionando que todos trabalhem os mesmos saberes, conhecimentos e competências essenciais. Para além disso, elas devem ser desafiantes, instigantes (na medida em que ajudem os alunos a pensar e a raciocinar para as poderem resolver) e apelativas para todos (TOMLINSON; IMBEAU, 2010).

Por último, não há diferenciação pedagógica sem uma organização de sala de aula flexível e facilmente modificável (UNESCO, 2004). De fato, numa sala de aula de ensino diferenciado é possível acontecer mais do que uma coisa ao mesmo tempo, o que representa a flexibilidade, mas que precisa de uma estrutura claramente definida para dar a estabilidade e a previsibilidade de que os professores e os alunos precisam. Nesse sentido, os professores podem desenvolver e implementar rotinas de sala de aula que permitam aos alunos trabalhar com sucesso individualmente, em grupo ou com toda a turma (SOUSA; TOMLINSON, 2018).

A este propósito, Tomlinson e Imbeau (2010) referem que os grupos de alunos que trabalham juntos devem ser frequentemente alterados (flexible grouping). O professor deve agrupar os alunos de acordo com os dados mais recentes de que dispõe a cada momento, por exemplo, se o professor agrupa os alunos de acordo com o seu nível de preparação, deve reorganizar os grupos a partir do momento em que se observa uma alteração no nível de preparação dos alunos. A flexibilidade e a variedade na organização dos grupos de alunos devem ser encorajadas, dando mesmo a possibilidade aos alunos de poderem escolher e mudar os seus grupos, de tempos a tempos. 


\section{MODELO DE DIFERENCIAÇÃO PEDAGÓGICA SEGUNDO TOMLINSON}

A diferenciação pedagógica é uma abordagem ao ensino em sala de aula que proporciona aos alunos múltiplas abordagens para o que vão aprender, como o vão fazer e como vão expressar o que aprenderam, a fim de maximizar as suas oportunidades de aprendizagem (TOMLINSON, 2008). Tem como elemento central a planificação ativa por parte do professor, focada nas diferentes necessidades dos alunos (FERREIRA, 2017), suficientemente flexível para se fazer as adaptações necessárias durante o processo de ensino-aprendizagem (PARSONS et al., 2013).

O modelo de diferenciação pedagógica concebido por Tomlinson (2008) centra-se na diferenciação de três componentes-chave do currículo, a saber, os conteúdos, os processos e os produtos, numa relação com o nível de preparação do aluno, com o seu interesse e o seu perfil de aprendizagem (Quadro 1). Embora seja mais fácil pensar em como diferenciar o ensino, examinando cada um desses componentes isoladamente, é importante ter consciência de que eles estão interligados e que, portanto, algumas decisões que o professor toma, em termos de elementos da planificação das aulas de ensino diferenciado, podem corresponder a dois ou mesmo a três componentes do currículo, pois "os alunos processam ideias à medida que leem o conteúdo, pensam ao mesmo tempo que criam produtos e pensam em ideias para produtos enquanto encontram ideias nos materiais usados" (TOMLINSON, 2008, p. 117).

QUADRO 1 - Modelo de diferenciação pedagógica

\begin{tabular}{|l|l|l|}
\hline Os professores podem diferenciar o \\
\hline Conteúdo & Processo & Produto \\
\hline $\begin{array}{l}\text { Informações, ideias e } \\
\text { competências que os } \\
\text { alunos vão interiorizar ou } \\
\text { desenvolver para atingir } \\
\text { os objetivos de } \\
\text { aprendizagem. }\end{array}$ & $\begin{array}{l}\text { quais os alunos dão } \\
\text { sentido às ideias-chave do } \\
\text { conteúdo, usando } \\
\text { conhecimentos e } \\
\text { competências essenciais. }\end{array}$ & $\begin{array}{l}\text { Como os alunos } \\
\text { demonstram o que sabem, } \\
\text { compreendem e são } \\
\text { capazes de fazer, em } \\
\text { resultado de uma unidade } \\
\text { ou de um conjunto de } \\
\text { aulas. }\end{array}$ \\
\hline De acordo com (dos seus alunos) & Perfil de Aprendizagem \\
\hline Nível de Preparação & Interesses & $\begin{array}{l}\text { Abordagens às } \\
\text { aprendizagens preferidas } \\
\text { dos alunos, influenciadas }\end{array}$ \\
\hline $\begin{array}{l}\text { Proximidade dos alunos a } \\
\text { objetivos de } \\
\text { aprendizagem }\end{array}$ & $\begin{array}{l}\text { As paixões pessoais e } \\
\text { situacionais dos alunos, } \\
\text { afinidades e }\end{array}$
\end{tabular}




\begin{tabular}{|c|c|c|}
\hline específicos. & $\begin{array}{l}\text { familiaridades que } \\
\text { motivam a aprendizagem. }\end{array}$ & $\begin{array}{l}\text { pelo estilo de } \\
\text { aprendizagem, inteligências } \\
\text { preferenciais, background } \\
\text { cultural e gênero. }\end{array}$ \\
\hline \multicolumn{3}{|c|}{ usando estratégias de ensino como } \\
\hline $\begin{array}{l}\text { Organizadores gráficos, } \\
\text { Tarefas por níveis, } \\
\text { Contratos e Agendas. }\end{array}$ & $\begin{array}{l}\text { Quebra-cabeças, } \\
\text { Menus de aprendizagem, } \\
\text { Centros de Interesse. }\end{array}$ & $\begin{array}{l}\text { IM (Inteligências Múltiplas), } \\
\text { Tarefas VARK, } \\
\text { Pontos de Entrada. }\end{array}$ \\
\hline \multicolumn{3}{|l|}{ tendo por base } \\
\hline \multicolumn{3}{|c|}{$\begin{array}{l}\text { Objetivos de aprendizagem comuns, } \\
\text { Avaliação diagnóstica e formativa, } \\
\text { Questionários de interesses e preferências. }\end{array}$} \\
\hline \multicolumn{3}{|c|}{ e implementadas através de } \\
\hline \multicolumn{3}{|c|}{$\begin{array}{l}\text { Grupos de trabalho variados, } \\
\text { Rotinas de sala de aula flexíveis, } \\
\text { Técnicas e ferramentas de gestão eficientes. }\end{array}$} \\
\hline \multicolumn{3}{|c|}{ no contexto de } \\
\hline
\end{tabular}

Fonte: Hockett, 2018 (adaptado, tradução livre).

O conteúdo é o que se ensina ou o que se quer que os alunos aprendam - input de ensino e aprendizagem, i.e., o que se quer que os alunos conheçam, compreendam e sejam capazes de fazer para atingir os objetivos de aprendizagem (HOCKETT, 2018; TOMLINSON; IMBEAU, 2010). Inclui fatos, conceitos, capacidades que os alunos vão adquirir no seu contexto de aprendizagem (UNESCO, 2004). A diferenciação do conteúdo pode passar pela utilização de uma variedade de materiais para a realização de uma mesma tarefa, com destaque para ideias-chave relacionadas a vida real, apresentados a partir de diversos métodos de ensino (e.g., verbal, visual, rítmico).

O processo corresponde às atividades de compreensão, ao modo como os alunos assimilam as ideias e a informação, i.e., ao modo como os alunos compreendem ou dão sentido ao conteúdo (TOMLINSON, 2008; TOMLINSON; IMBEAU, 2010). É importante que as atividades estejam alinhadas com os objetivos do conteúdo, que sejam meios efetivos de aprendizagem, focadas num aspecto essencial de algo que os alunos precisam conhecer, compreender e serem capazes de fazer, que "os ajude a «possuir» o conteúdo, a ver como ele faz sentido e a perceber a sua utilidade no mundo fora da sala de aula" (TOMLINSON; IMBEAU, 2010, p. 15, tradução livre). A diferenciação do processo pode incluir a escolha de 
trabalhar em grupo ou individualmente, o uso flexível do tempo, os contratos de aprendizagem, entre outros.

O produto designa o modo como os alunos demonstram o que vieram a saber, o que vieram a compreender e o que são capazes de fazer depois de um certo período de aprendizagem (TOMLINSON; IMBEAU, 2010). Se as atividades de compreensão são, no geral, breves e centradas num ou apenas em alguns conhecimentos e capacidades essenciais, "um produto é um esforço a longo prazo" (TOMLINSON, 2008, p. 135). Desse modo, representam os vastos conhecimentos e aplicações dos alunos, sendo a estrutura dos testes um bom exemplo disso. A diferenciação do produto pode envolver, por exemplo, vários modos de expressão das aprendizagens dos alunos (e.g., apresentação oral, debate, exposição, dramatização), a definição de objetivos pessoais, providenciar amostras de bom trabalho dos alunos com diferentes níveis de complexidade.

Embora em 2008, data de referência para a apresentação desse modelo de diferenciação pedagógica, Tomlinson tenha referido apenas o conteúdo, o processo e o produto como os componentes-chave do currículo que o professor pode diferenciar (TOMLINSON, 2008), mais tarde (TOMLINSON; IMBEAU, 2010), introduz um outro elemento, o afeto, para dar conta de como as emoções e os sentimentos dos alunos influenciam as suas aprendizagens, i.e., de como a sua motivação para aprender, a sua habilidade para trabalhar com os pares e a sua autoestima como aluno está dependente das emoções e sentimentos gerados pelas experiências passadas e pelas reações às presentes, sendo, por isso, parte integrante do currículo. Nesse sentido, cabe, também, aos professores trabalharem para compreender essas emoções e sentimentos e poderem adaptar o ambiente de aprendizagem, de forma a guiarem os alunos numa direção positiva (TOMLINSON; IMBEAU, 2010).

Vários estudos indicam que um ensino que responda ao nível de preparação do aluno (capacidades e compreensão sobre um determinado tema), ao seu interesse (curiosidade ou paixão) ou ao seu perfil de aprendizagem (maneira preferida de trabalhar) está associado a resultados 
mais apropriados à diversidade dos alunos e a um melhor desempenho dos mesmos (e.g., JARVIS et al., 2017; TOMLINSON et al., 2003).

A preocupação com o nível de preparação do aluno teve a sua origem no conceito vigotskyano de 'zona de desenvolvimento proximal' (CIVITILLO et al., 2016; JARVIS et al., 2017; TOMLINSON et al., 2003), advertindo para a questão dos alunos trabalharem em níveis moderados de dificuldade, pois mais facilmente mantêm os esforços para aprender (BRANSFORD, BROWN; COCKING, 2000), sem se sentirem entediados ou enganados, quando as tarefas são demasiado fáceis, ou ansiosos e frustrados, quando as tarefas apresentadas estão muito além das competências existentes (JARVIS et al., 2017). Para abordar efetivamente os níveis de preparação dos alunos, os professores devem questionar-se sobre o grau de correspondência entre o nível atual de conhecimento, compreensão e capacidade do aluno e o que ele é chamado a realizar a cada momento (SOUSA; TOMLINSON, 2018), i.e., o aluno está em que momento específico do seu processo educativo e, de acordo com os objetivos de aprendizagem, em que momento ele deveria estar (HOCKETT, 2018).

É importante não confundir o nível de preparação de um aluno, que sugere uma condição temporária, que deve mudar regularmente, com as suas capacidades que são, geralmente, conotadas com uma característica mais ou menos fixa e inata (TOMLINSON; IMBEAU, 2010). O professor pode recolher informação sobre o nível de preparação dos seus alunos por meio de uma avaliação de diagnóstico, antes de iniciar uma unidade ou um conjunto de aulas (e.g., estratégia KWL e Concordo ou discordo?); por meio de uma avaliação formativa/contínua, que permite ao professor ir fazendo os ajustes necessários à sua planificação (e.g., questionários rápidos (Quick Quizzes), lembretes (Sticky Notes), sumários de um tópico, brainstorming).

Para além da adequação do ensino ao nível de preparação do aluno, o seu interesse e a sua motivação para aprender são também importantes para o seu desenvolvimento acadêmico. Na revisão de literatura, apresentada por Tomlinson e colaboradores (2003), é referido que adequar o ensino ao interesse do aluno tem impactos positivos na sua aprendizagem, 
aumentando a sua motivação, a sua produtividade e a sua realização, e que há uma relação entre o cumprimento de tarefas que os alunos têm curiosidade e o seu envolvimento e empenho nas mesmas (TOMLINSON, 2008). Assim, adequar o ensino ao interesse dos alunos torna-os, cognitiva e afetivamente, mais envolvidos com a aprendizagem (HOCKETT, 2018), estando os interesses pessoais "geralmente ligados aos seus pontos fortes, ao seu contexto cultural, às experiências pessoais, às questões que colocam ou ao sentido de necessidade que têm" (TOMLINSON; IMBEAU, 2010, p. 17, tradução livre). O professor pode utilizar várias estratégias para conhecer os interesses dos seus alunos como, por exemplo: os grupos jigsaw, os Menus de Aprendizagem, as entrevistas a pares, os inventários de interesses.

O perfil de aprendizagem do aluno descreve o modo como o aluno aprende melhor, como processa o que precisa aprender ou como pensa, recorda e prefere usar o que aprende (SOUSA; TOMLINSON, 2018). Por exemplo, enquanto certos alunos aprendem mais facilmente sozinhos, outros preferem aprender em grupo. Neste sentido, o perfil de aprendizagem do aluno é mais um conjunto de preferências do que um conjunto de características ou traços inerentes ao próprio aluno, i.e., não deve servir para categorizar os alunos em tipo de aluno $X$ ou $Y$, até porque a maioria das pessoas aprende de maneiras muito diversas (HOCKETT, 2018).

A pesquisa mostrou que há quatro dimensões sobrepostas ou elementos interligados (Figura 2) que influenciam o perfil de aprendizagem do aluno, a saber, o estilo de aprendizagem, as inteligências preferenciais, a cultura e o gênero (HOCKETT, 2018; TOMLINSON et al., 2003; TOMLINSON, 2008; TOMLINSON; IMBEAU, 2010). Os alunos aprendem de maneiras diferentes e aprendem de modo mais eficaz quando as circunstâncias da aprendizagem coincidem com a sua abordagem preferida (HOCKETT, 2018). Do mesmo modo, um ensino que contemple os pontos fortes dos alunos nas diferentes áreas da sua inteligência ou modos de pensar, como sugerem, por exemplo, a Teoria das Inteligências Múltiplas de Howard Gardner (1995) e a Teoria Triárquica da Inteligência de Robert Sternberg (1985), resulta num melhor desempenho dos alunos (TOMLINSON, 2008). Para além disso, a 
pesquisa indica que a cultura e o gênero influenciam o modo como se aprende (SOUSA; TOMLINSON, 2018; TOMLINSON, 2008; TOMLINSON; IMBEAU, 2010), i.e., moldam a predisposição do aluno para, por exemplo, trabalhar autonomamente ou em colaboração, para interpretar a informação literalmente ou em sentido figurativo, para abordar as tarefas de forma ordenada ou de forma indireta (HOCKETT, 2018).

FIGURA 2 - Fatores para Perfis de Aprendizagem

\begin{tabular}{|l|l|}
\hline Orientação do Grupo & $\begin{array}{l}\text { Ambiente de Aprendizagem/Contexto } \\
\text { Educativo }\end{array}$ \\
\hline $\begin{array}{l}\text { Auto-orientação/autônoma, } \\
\text { Orientação de grupo/pares, } \\
\text { Orientação de adultos mista. }\end{array}$ & $\begin{array}{l}\text { Sossegado/barulhento, } \\
\text { Quente/fresco, } \\
\text { Imóvel/móvel, } \\
\text { Flexível/fixo. }\end{array}$ \\
\hline Estilo Cognitivo & Inteligência Preferencial \\
\hline & Analítica, \\
Criativo/conformista, & Prática, \\
Essência/fatos, & Criativa, \\
Expressivo/controlado, & Verbal-linguística, \\
Concreto/abstrato, & Lógico-matemática, \\
Colaborativo/competitivo, & Visual-espacial, \\
Oral/visual/cinestésico, & Corporal-cinestésica, \\
Reflexivo/orientado para a ação, & Musical-rítmica, \\
Facilmente distraído/índice elevado de & Interpessoal, \\
atenção. & Intrapessoal, \\
& Naturalista. \\
\hline
\end{tabular}

Fonte: Tomlinson, 2008 (adaptado).

De acordo com Hockett (2018), o professor pode aferir o perfil de aprendizagem dos alunos por meio de perguntas sobre as suas preferências, observando como os alunos trabalham e as escolhas que fazem, muito à semelhança do que acontece quando pretende "avaliar" o interesse dos alunos. Algumas estratégias para recolher essa informação são Pontos de Entrada (Entry Points), Mente Tripartida (TriMind) e um questionário VARK.

\section{DIFERENCIAÇÃO PEDAGÓGICA INCLUSIVA: potencialidades e barreiras}

A premissa base da diferenciação pedagógica, não sendo nova, tem sido alvo de uma maior atenção nas últimas duas décadas (ROCK et al., 2008). Inúmeros estudos têm vindo a demonstrar o seu efeito positivo no desempenho dos alunos, quer em termos acadêmicos, quer em termos 
pessoais (ROCK et al., 2008; TIESO, 2002; TOMLINSON et al., 2003; WHIPP; TAGGART; JACKSON, 2014).

Baumgartner e colaboradores (2003), num estudo com base na implementação de um programa de leitura assente em estratégias de diferenciação pedagógica, apresentam dados que evidenciam melhorias nos resultados da leitura expressos em um aumento das estratégias de compreensão utilizadas pelos alunos, em um maior domínio das competências fonêmicas e de descodificação, nas atitudes dos alunos face à leitura e mesmo nas suas perceções sobre as competências próprias de cada um para a leitura. No mesmo sentido, Santamaria (2009), focando-se na aprendizagem do inglês por alunos do ponto de vista linguístico culturalmente diferentes, observou que as práticas de ensino mais eficazes são aquelas que consideram todos os alunos em sala de aula e prestam atenção à diversidade acadêmica, cultural, linguística e socioeconômica dos alunos. Por seu lado, Tieso (2002) examinou os efeitos de práticas curriculares diferenciadas nos resultados dos alunos em matemática. Num desenho quasi-experimental com uma amostra aleatória estratificada de 31 professores e os seus alunos ( $n=645$ ), de quatro distritos escolares da Nova Inglaterra, Tieso (2002) revelou que os alunos, alvo das práticas curriculares diferenciadas, demonstraram pontuações mais altas e melhorias no seu desempenho quando comparados com os alunos não sujeitos a essas práticas.

Contudo, apesar das evidências positivas da utilização de práticas de diferenciação pedagógica na sala de aula, poucos professores as implementam (CIVITILLO et al., 2016; PRUD'HOMME et al., 2005; TOMLINSON et al., 2003; TOMLINSON, 2016), sobretudo em razão de barreiras relacionadas às suas concepções e crenças (e.g., sobre educação, currículo, ensino, diversidade); aos aspectos organizacionais da escola (e.g., tempo, recursos, apoios); às pressões da própria escola e dos pais; e aos aspectos relativos às lideranças.

No tocante às concepções e crenças dos professores como barreiras ao desenvolvimento da diferenciação pedagógica, vários autores 
destacam a falta de clarificação do conceito de diferenciação pedagógica (FEYFANT, 2016; PRUD'HOMME et al., 2016). A esse propósito, Tomlinson (2016) identifica um conjunto de crenças que se constituem como barreiras ao desenvolvimento de práticas de diferenciação pedagógica, e.g., pensar que o ensino é transmissão; que aprender é absorver e reproduzir; que os alunos são majoritariamente dependentes e incapazes de independência; que gerir é sinônimo de controlar; que algumas crianças são capazes e outras não; que o justo é tratar todos da mesma maneira; que o currículo é sobretudo dar a matéria toda; que aprender é repetir informações e resolver exercícios; que a avaliação é realizada no final para ver quem percebeu e para nivelar os alunos (TOMLINSON, 2016). Outros estudos evidenciam crenças de autoeficácia baixa para desenvolver esse tipo de prática. Como refere Dixon e colaboradores (2014), o esforço exigido para ser flexível e adaptar as aulas às necessidades dos alunos é sentido pelos professores como sendo demasiado para eles.

Para além disso, o modo como os professores concebem a diversidade é também uma barreira à implementação da diferenciação pedagógica em sala de aula (DIXON et al., 2014; JAGER, 2013). De fato, perspectivar a diversidade em sala de aula como um desafio é facilitador dessa implementação, do mesmo modo que a conceber como um problema é um obstáculo (JAGER, 2013). Nessa linha de ideias, Dixon e colaboradores (2014) salientam a dificuldade que representa para os professores compreender que a diversidade de aprendizagens presente na sala de aula pode garantir o sucesso de todos os alunos.

Estudos recentes sugerem que, muito embora os professores manifestem crenças mais positivas em relação à importância da diferenciação pedagógica, persistem algumas dificuldades na transposição de novos conhecimentos e crenças para as práticas. Assim, os professores reconhecem a diversidade dos alunos nas salas de aula e frequentemente afirmam a necessidade de lidar com as suas diferenças para os fazer atingir o sucesso (FEYFANT, 2016; HEHIR et al., 2016; TOMLINSON et al., 2003; TOMLINSON, 2016). Contudo, a sua prática tende a ser desalinhada com 
essas crenças (TOMLINSON, 2016; MAIA; FREIRE, 2019, submetido), sendo que alguns professores revelam dificuldade em transferir essa necessidade para a prática na sala de aula (FEYFANT, 2016).

Outros autores têm vindo a realçar a falta de preparação/formação dos professores como um outro obstáculo ao desenvolvimento da diferenciação pedagógica em sala de aula (e.g., DIXON et al., 2014; HEHIR et al., 2016; JAGER, 2013; WAN, 2017). De acordo com Wan (2017), os professores não estão suficientemente preparados, em termos de conhecimento processual e pedagógico, para orientar aulas de ensino diferenciado.

Em termos organizacionais, uma barreira frequentemente mencionada é a falta de tempo dos professores (e.g., CIVITILLO et al., 2016; JAGER, 2013; NAZZAL, 2011), que resulta do aumento da sua carga de trabalho, das sucessivas mudanças curriculares, das atividades extracurriculares em que têm de participar e das formações em horário pós-laboral (JAGER, 2013). A par da falta de tempo dos professores, surge a necessidade de estes trabalharem colaborativamente, dado que os estudos indicam que 0 envolvimento do professor em comunidades de aprendizagem profissional influencia positivamente a prática diferenciada em salas de aula (WAN, 2017). Para além disso, a colaboração pressupõe uma mudança de papéis dos professores, i.e., o professor como transmissor de conhecimento dá lugar ao professor como facilitador do mesmo, o que Ihes coloca maiores exigências no trabalho (WAN, 2017).

A falta de recursos e de apoios é, em termos organizacionais, outro fator mencionado como barreira à implementação da diferenciação pedagógica em sala de aula (CIVITILLO et al.; 2016; JAGER, 2013; TOMLINSON, 2016). Segundo Jager (2013), a falta de recursos continua a ser uma desvantagem para atender às diferentes necessidades dos alunos e para implementar atividades de aprendizagem diferenciada. Para além disso, a falta de espaço (NAZZAL, 2011), as turmas numerosas (CIVITILLO et al., 2016; JAGER, 2013), as dificuldades na gestão da sala de aula e do comportamento dos alunos (NAZZAL, 2011; ROCK et al., 2008) têm também 
sido apontados como barreiras ao desenvolvimento da diferenciação pedagógica. Finalmente, há ainda a referir a questão do currículo, tal como interpretado pelos professores, e as dificuldades que esse coloca à diferenciação pedagógica. Com efeito, alguns estudos identificam como obstáculo a prioridade dada pelos professores ao cumprimento do programa disciplinar em detrimento da diferenciação pedagógica (CIVITILLO et al., 2016; NAZZAL, 2011; ROCK et al., 2008) ou a tensão que esse cumprimento exerce nos professores quando reinterpretam o programa em função das necessidades dos alunos (FEYFANT, 2016).

Uma outra barreira ao desenvolvimento de práticas de diferenciação pedagógica em sala de aula diz respeito às influências e pressões que a escola enquanto instituição exerce nos professores, e.g., o modo como os prepara para ensinar, para supervisionar ou para 'controlar' outros professores (TOMLINSON, 2016). Para além disso, o fato de poucos professores terem experiência pessoal, aprendendo ou ensinando, em salas de aula diferenciadas, i.e., em salas em que o aluno está no centro da tomada de decisões, constitui-se, também, uma barreira à implementação da diferenciação pedagógica, tal como a visão de liderança da escola e os próprios pais, ao preferirem estruturas educacionais e abordagens que thes sejam familiares e que percebam ser vantajosas para os seus filhos (TOMLINSON, 2016).

\section{CONCLUSÃO}

Não é possível ao professor, nos dias de hoje, encarar uma turma como se os seus alunos fossem, essencialmente, parecidos e para quem, com a mesma aula, fosse possível maximizar a participação e sucesso de todos (TOMLINSON, 2008). De fato, há inúmeras evidências que uma aula tradicional, majoritariamente expositiva, ancorada numa abordagem transmissiva e uniformizada do ensino, resulta em desigualdades no atendimento às diferenças de todos os alunos (JAGER, 2013; TOMLINSON, 2016). 
A diferenciação pedagógica inclusiva surge como uma abordagem ao ensino que procura assegurar para todos os alunos, independentemente das suas diferenças, sucesso na aprendizagem (FEYFANT, 2016; UNESCO, 2017). Contudo, a investigação revela que os professores tendem a implementá-la pouco ou os que a aplicam não o fazem de forma refletida e consistente, em grande parte, porque a diferenciação pedagógica não é bem entendida pelos professores (HOCKETT, 2018). Nesse sentido, torna-se imperioso clarificar o que se entende por diferenciação pedagógica para que, dessa forma, se promova o seu desenvolvimento nas salas de aula.

Vários autores que procuraram esclarecer esse conceito (HOCKETT, 2018; SANCHES, 2005; TOMLINSON; IMBEAU, 2010; PRUD'HOMME et al., 2016; TOMLINSON, 2008) sentiram necessidade de, primeiramente, desconstruir alguns equívocos que se tendem a perpetuar no discurso dos professores e que contribuem para a imprecisão e confusão em torno desse conceito. Assim, convém relembrar que a diferenciação pedagógica não é uma moda educacional que acabará por desaparecer, nem um conjunto de estratégias de ensino que algum especialista pode demonstrar como se aplicam, tão pouco a ideia de que ela não possibilita o ensino para toda a turma, sendo mais fácil em alguns ciclos ou matérias do que em outros.

Com efeito, a diferenciação pedagógica inclusiva é uma abordagem ao ensino que pressupõe uma planificação proativa por parte do professor para responder, de forma consistente, ao nível de preparação, interesse e perfil de aprendizagem dos seus alunos, diferenciando o conteúdo, o processo e o produto (TOMLINSON, 2008). É um processo individual (não há receitas!) que resulta de o professor ir tentando implementá-la, de refletir sobre essas tentativas e de fazer os ajustamentos necessários na própria sala de aula (TOMLINSON; IMBEAU, 2010). Planificar um ensino diferenciado é como construir diferentes roteiros (flexibilidade curricular) para uma mesma visita de estudo (objetivos de aprendizagem) ou como ser o gestor de um restaurante (o professor) e ter à disposição de todos os clientes (alunos) um menu variado (conteúdo, processo e produto diferenciados), que satisfaça os diferentes gostos (perfil de aprendizagem). 
Assim, importa pensar que contributo é possível dar para que os professores analisem e reflitam sobre as suas práticas e eventualmente as mudem. A literatura dá-nos algumas pistas para isso. Vários estudos apontam para a relevância que o processo de desenvolvimento profissional tem na mudança educacional, tendo em vista a melhoria no resultado dos alunos (ANTONIOU; KYRIAKIDES, 2013; DRITS-ESSER et al., 2017; ZAMBAK et al., 2017), e, em particular, a UNESCO (2017) que recomenda que os programas de formação capacitem e apoiem os professores no desenvolvimento de uma gama mais ampla de respostas para os alunos com dificuldades na sua aprendizagem. Nesse contexto, é preciso apostar no desenvolvimento profissional dos professores, em especial, na sua formação, pois a falta dela é, também, apontada como uma barreira ao desenvolvimento de práticas de diferenciação pedagógica. Devem privilegiar-se ambientes de formação, formais ou informais, propiciadores de reflexão, de partilha de ideias com foco na dimensão pedagógica da ação docente (e.g., grupos de trabalho de pequena dimensão, colóquios, seminários, observação interpares). Como refere Girardet (2018), os programas de desenvolvimento profissional parecem mais eficazes na promoção da mudança educacional quando: (i) incluem atividades focadas na reflexão sobre crenças prévias dos professores; (ii) integram o estudo de práticas alternativas e o modo como pô-las em prática e (iii) envolvem a reflexão em ação, num ambiente de aprendizagem colaborativo.

\section{REFERÊNCIAS}

AINSCOW, M.; BOOTH, T. Índex para a inclusão: desenvolvendo a aprendizagem e a participação na escola. Sintra: Cidadãos do Mundo, 2002.

AINSCOW, M. Tornar a educação inclusiva: como essa tarefa deve ser conceituada? In: FAVERO, O.; FERREIRA, W. (Org.). Tornar a Educação Inclusiva. Brasília: UNESCO, 2009. p. 11-24.

ANDREW, L. Comparison of teacher educators' instructional methods with the constructivist ideal. The Teacher Educator, v. 42, n. 3, 2007. p. 157-184.

ANTONIOU, P.; KYRIAKIDES, L. A dynamic integrated approach to teacher professional development: impact and sustainability of the effects on 
improving teacher behaviour and student outcomes. Teaching and teacher education, n. 29, 2013. p. 1-12.

BAUMGARTNER, T.; LIPOWSKI, M.; RUSH, C. Increasing reading achievement of primary and middle school students through differentiated instruction. 2003. Unpublished doctoral dissertation. Saint Xavier University, Chicago, 2003.

BRANSFORD, J.; BROWN, A.; COCKING, R. How people learn: mind, brain, experience, and school. Washington, DC: National Academy Press, 2000.

CIVITILLO, S.; DENESSEN, E.; MOLENAAR, I. How to see the classroom through the eyes of a teacher: consistency between perceptions on diversity and differentiation practices. Journal of Research in Special Educational, v 16, n. s1, 2016. p. 587-591.

CORREIA, L. Educação inclusiva ou educação apropriada?. In: RODRIGUES, D. (Org.). Educação e Diferença. Valores e Práticas para uma Educação Inclusiva. Porto: Porto Editora, 2001. p. 123-142.

CORNO, L.; SNOW, R. Adapting teaching to individual differences among learners. In: WITTROCK, M. C. (Ed.). Handbook of research on teaching. New York: Macmillan, 1986. p. 606-629.

DIXON, F. A.; YSSEL, N.; MCCONNELL, J. M.; HARDIN, T. Differentiated Instruction, Professional Development, and Teacher Efficacy. Journal for the Education of the Gifted, 2014. p. 1-17.

DRITS-ESSER, D.; GESS-NEWSOME, J.; STARK, L. A. Examining the sustainability of teacher learning following a year-long science professional development programme for inservice primary school teachers. Professional Development in Education, v 43, n. 3, p. 375-396, 2017.

FARRELL, P. The impact of research on developments in inclusive education. International Journal of Inclusive Education, v 4, n. 2, p. 153-162, 2000.

FEYFANT, A. La différenciation pédagogique en classe. Dossier de veille de I'IFÉ, n. 113. Lyon: ENS de Lyon, 2016.

FERREIRA, M. Guia para uma Pedagogia Diferenciada em Contexto de Sala de Aula: teorias, práticas e desafios. Lisboa: Coisas de Ler Edições, 2017.

GARDNER, H. Inteligências Múltiplas A Teoria na Prática. Porto Alegre: Artes Médicas, 1995.

GIRARDET, C. Why do some teachers change and others don't? A review of studies about factors influencing in-service and pre-service teachers' change in classroom management. Review of Education, v 6, n. 1, p. 3-36, 2018. 
HEHIR, T.; GRINDAL, T.; FREEMAN, B.; LAMOREAU, R.; Borquaye, Y.; Burke, S. Os Benefícios da Educação Inclusiva para Estudantes com e sem Deficiência. São Paulo: Instituto Alana, 2016.

HOCKETT, J. A. Differentiation Strategies and Examples: Grades 6-12. Tennessee: Department of Education, 2018.

HUMPHREY, N.; BARTOLO, P.; ALE, P.; CALLEJA, C.; HOFSAESS, T.; JANIKOVA, V.; WESTO, G. M. Understanding and responding to diversity in the primary classroom: An international study. European Journal of Teacher Education, $v$ 29, n. 3, p. 305-318, 2006.

JAGER, T. Guidelines to assist the implementation of differentiated learning activities in South African secondary schools. International Journal of Inclusive Education, v 17, n. 1, p. 80-94, 2013.

JARVIS, J. M.; PILL, S. A.; NOBLE, A. G. Differentiated Pedagogy to Address Learner Diversity in Secondary Physical Education. Journal of Physical Education, v 88, n. 8, p. 46-54, 2017.

KALDI, S.; GOVARIS, C.; FILIPPATOU, D. Teachers' views about pupil diversity in the primary school classroom. Compare: a Journal of Comparative and International Education, v 48, n. 1, 2-20, 2018.

LATORRE, L. V. La educación inclusiva en docentes en formación: su evaluación a partir de la teoría de facetas. FOLIOS, segunda época, n. 37, p. 95-113, 2013.

LIMA-RODRIGUES, L. (Coord.). Percursos de Educação Inclusiva em Portugal: dez estudos de caso. Lisboa: Faculdade de Motricidade Humana, 2007.

MAIA, V.; FREIRE, S. (submetido) Crenças e práticas dos professores em relação à diferenciação pedagógica. In.: Atas do IV Encontro Luso-Brasileiro Trabalho Docente e Formação de Professores. Lisboa: Instituto de Educação da Universidade de Lisboa e Universidade Federal de Alagoas, 2019.

MEIRIEU, P. Pédagogie: des lieux communs aux concepts-clés. Paris: ESF éditeur, 2013.

NAZZAL, A. Differentiation in Practice: An Exploration of First Year Teacher Implementation of Differentiation Strategies as Expected Outcomes of Teacher Preparation Program. Current Issues in Middle Level Education, v 16 , n. 1, p. 17-27, 2011.

PARSONS, S. A.; DODMAN, S. L.; BURROWBRIDGE, S. C. Broadening the view of differentiated instruction. kappanmagazine.org, $\vee 95, n$. 1, p. 38-42, 2013.

PORTUGAL. Decreto-Lei $\mathbf{n}^{\circ}$. 54, de 6 de julho de 2018. 
PRUD'HOMME, L.; DOLBEC, A.; BRODEUR, M.; PRESSEAU, A.; MARTINEAU, S. La construction d'un îlot de rationalité autour du concept de différenciation pédagogique. Journal of the Canadian Association for Curriculum Studies, $v$ 3, n. 1, p. 1-31, 2005.

PRUD'HOMME, L.; PARÉ, M.; LEBLANC, M.; BERGERON, G.; DESSEMONTET, R. S.; NOËL, I. La différenciation pédagogique dans une perspective inclusive : quand les connaissances issues de la recherche rencontrent le projet d'éducation pour tous. In: PRUD'HOMME, L. ; DUCHESNE, H.; BONVIN, P.; VIENNEAU, R. (Dirs.). L'inclusion scolaire: ses fondements, ses acteurs et ses pratiques. Louvain-la-Neuve: De Boeck Supérieur, 2016. p. 123-137.

PRZESMYCKI, H. Pédagogie Différenciée. Paris : Hachette Éducation, 1991.

RICHARDSON, V. Preservice teachers' beliefs. In: RATHS, J.; MCANINCH, A. (Eds.). Teacher beliefs and classroom performance: the impact of teacher education. Charlotte, NC: Information Age Publishing, 2003. p. 1-22.

ROBBES, B. La pédagogie différenciée : historique, problématique, cadre conceptuel et méthodologie de mise en œuvre. La pédagogie différenciée, p. 1-34, 2009.

ROCK, M. L.; GREGG, M.; ELLIS, E.; GABLE, R. A. REACH: A framework for differentiating classroom instruction. Preventing School Failure, v 52, n. 2, p. 31 47, 2008.

SANCHES, I. Compreender, Agir, Mudar, Incluir. Da investigação-acção à educação inclusiva. Revista Lusófona de Educação, n. 1, p.127-142, 2005.

SANCHES, I.; TEODORO, A. Procurando indicadores de educação inclusiva: as práticas dos professores de apoio educativo. Revista Portuguesa de Educação, v 20, n. 2, p. 105-149, 2007.

SÁNCHEZ, P. A. A educação inclusiva na Espanha. In: FAVERO, O.; FERREIRA, W. (Org.). Tornar a Educação Inclusiva. Brasília: UNESCO, 2009. p. 89-104.

SANTAMARIA, L. J. Culturally responsive differentiated instruction: Narrowing the gaps between best pedagogical practices for all learners. Teachers College Record, v 111, n. 1, p. 214-247, 2009.

SORIANO, V.; HUGHES, G. (eds.). Promoting Common Values and Inclusive Education: Reflections and Messages. European Agency for Special Needs and Inclusive Education. Dinamarca: Odense, 2018.

SOUSA, F. Diferenciação Curricular e Deliberação Docente. Porto: Porto Editora, 2010. 
SOUSA, D.; TOMLINSON, C. Differentiation and the brain. How Neuroscience Supports the Learner-Friendly Classroom. (2nd ed). Bloomington, IN: Solution Tree Press, 2018.

STERNBERG, R. Beyond IQ A triarchic theory of human intelligence. Cambridge University Press, 1985.

TIESO, C. The Effects of Grouping Practices and Curricular Adjustments on Achievement. Journal for the Education of the Gifted, v 29, n. 1, P. 60-89, 2002.

TOMLINSON, C. A.; BRIGHTON, C.; HERTBERG, H.; CALLAHAN, C. M.; MOON, T. R.; BRIMIJOIN, K.; CONOVER, L. A.; REYNOLDS, T. Differentiating instruction in response to student readiness, interest, and learning profile in academically diverse classrooms: A review of the literature. Journal for the Education of the Gifted, n. 27, p. 119-145, 2003.

TOMLINSON, C. A. Diferenciação Pedagógica e Diversidade - Ensino de Alunos em Turmas com Diferentes Níveis de Capacidades. Coleção Educação Especial. Porto: Porto Editora, 2008.

TOMLINSON, C. A.; IMBEAU, M. B. Leading and Managing A Differentiated Classroom. Alexandria, VA: ASCD, 2010.

TOMLINSON, C. A. The Differentiated Classroom: responding to the Needs of All Learners. (2nd ed). Alexandria, VA: ASCD, 2014.

TOMLINSON, C. A. Why differentiation is difficult: Reflections from years in the trenches. Australian Educational Leadership, v 38, n. 3, p. 6-8, 2016.

UNESCO. Changing Teaching Practices using curriculum differentiation to respond to students' diversity. Paris: UNESCO, 2004.

UNESCO. Guidelines For Inclusion: Ensuring Access to Education for All. Paris: UNESCO, 2005.

UNESCO. Directrices sobre políticas de inclusión en la educación. Paris: UNESCO, 2009.

UNESCO. A guide for ensuring inclusion and equity in education. Paris: UNESCO, 2017.

WAN, S. Differentiated instruction: are Hong Kong in-service teachers ready?. Teachers and Teaching, $v$ 23, n. 3, p. 284-311, 2017.

WHIPP, P.; TAGGART, A.; JACKSON, B. Differentiation in outcome focused physical education: Pedagogical rhetoric and reality. Physical Education and Sport Pedagogy, n. 19, p. 370-382, 2014. 
ZAMBAK, V. S.; ALSTON, D. M.; MARSHALL, J. C.; TYMINSKI, A. M. Convincing Science Teachers for Inquiry-Based Instruction: Guskey's Staff Development Model Revisited. Science Educator, v 25, n. 2, 108-1 16, 2017.

Recebido em: 30 de junho de 2019 Aprovado em: 25 de novembro de 2019 